\title{
Cultivating Moina macrocopa Straus in different media using carotenogenic yeast Rhodotorula
}

\author{
Olga Kushniryk, Oleksii Khudyi, Lidiia Khuda, Ryszard Kolman, Mykhailo Marchenko
}

Received - 21 October 2014/Accepted - 20 January 2015. Published online: 31 March 2015; ๑Inland Fisheries Institute in Olsztyn, Poland Citation: Kushniryk O., Khudyi O., Khuda L., Kolman R., Marchenko M. 2015 - Cultivating Moina macrocopa Straus in different media using carotenogenic yeast Rhodotorula - Arch. Pol. Fish. 23: 37-42.

\begin{abstract}
The possibility of using carotenogenic yeast from the genus Rhodotorula as food substrate was studied during the cultivation of Moina macrocopa. The results showed that replacing traditional food substrates during the cultivation of the live fish food $M$. macrocopa resulted in improved characteristics that were superior to the alternative. Using carotenogenic yeast Rhodotorula glutinis (Fres.) Harrison to stimulate carotenoid accumulation in live feed is better than $R$. rubra (Demme) Lodder. The optimal duration of enriching M. macrocopa with carotenoids was four days when using various types of yeast capable of carotenogenesis. Replacing the standard medium for culturing $M$. macrocopa with the alternative medium was less expensive. Breeding live feed on recirculating aquaculture system (RAS) post-production water was proven to be effective.
\end{abstract}

Keywords: carotenoid sources, culture media, live feed, Cladocera

O. Kushniryk, O. Khudyi [ $\Xi^{\circ}$ ], L. Khuda, M. Marchenko Department of Biochemistry and Biotechnology Yuriy Fedkovych Chernivtsi National University, Ukraine e-mail: khudij@email.ua

R. Kolman

Department of Ichthyology

Inland Fishery Institute in Olsztyn, Poland

\section{Introduction}

Since fish, like all animals, cannot synthesize carotenoids, these pigments must be supplied to them exogenously by organisms that are capable of carotenogenesis, such as algae, yeast, and some bacteria (Buzzini and Martini 1999, Gupta et al. 2007, Guedes et al. 2011, Nechypurenko et al. 2013). More than 20 carotenoids have been identified in fish, the most common of which are astaxanthin, canthaxanthin, $\beta$-carotene, and lutein (Czeczuga et al. 1991). These pigments are concentrated in the muscles, liver, integuments, fins, intestinal walls, and gonads (Czeczuga et al. 1991, Wozniak 1996, Bjerkeng 2000). Carotenoids in fish can be transdifferentiated as required by particular organisms; the rare carotenoid idoxanthyn, which is found in the skin and muscle tissue of bitterling males (Czeczuga 1994), is an intermediate product of $\beta$-carotene transdifferentiation into astaxanthin (Czeczuga 1988). The content of carotenoids and their distribution in fish is species specific and depends on gender, age, and physiological condition (Czeczuga 1994).

The qualitative composition and quantitative content of carotenoids in fish depend on their content in feed. Since fish are better able to absorb live feed than granulated, it is particularly effective to use live feed as a starter (Faruque et al. 2010, Das et al. 2012). Accordingly, the technology of carotenoid

\footnotetext{
(c) Copyright by Stanisław Sakowicz Inland Fisheries Institute in Olsztyn.

(C) 2015 Author(s). This is an open access article licensed under the Creative Commons Attribution-NonCommercial-NoDerivs License (http://creativecommons.org/licenses/by-nc-nd/3.0/).
} 
saturation in organisms used in aquaculture and aquarium husbandry as live feed is relevant, as planktonic crustaceans are the traditional source of live feed.

Increasing the carotenoid content of live feed with carotenogenic yeast is a cheaper alternative to using expensive commercial carotenoid complex products. Additional savings can be made by reducing the technological costs of culturing live feed by using other types of culture media that do not require large expenditures. During fish propagation in recirculating aquaculture systems (RAS), the water has to be replaced periodically and the post-production water can be used as a cheaper alternative to the traditional media used in aquatic organism cultivation. Consequently, live feed was cultivated with either RAS post-production water or a traditional medium and two types of carotenogenic yeasts. The aim of the study was to assess the possibility of using carotenogenic yeast as a food substrate and source of carotenoids for M. macrocopa and to evaluate RAS post-production water for use in live feed cultivation.

\section{Materials and methods}

The experiment was conducted using pure cultures of M. macrocopa. The crustaceans were cultivated with an initial number of individuals of 50 ind. $1^{-1}$ in $0.5 \mathrm{l}$ volumes with a 16 -hour photoperiod, and at a temperature of $22 \pm 1^{\circ} \mathrm{C}$ in climatic chamber conditions according to established procedures (Romanenko et al. 1999). The research organisms were cultivated in a synthetic ADaM meduim (Kluttgen et al. 1994) and in post-production water obtained from a RAS mechanical filter.

Aqueous suspensions of the yeasts Saccharomyces cerevisiae, $R$. glutinis, and R. rubra were used as the food substrate for the zooplankton. Six experimental groups were created with the food substrates and cultivation media (Table 1). The experiment was conducted in nine replicates. Organisms cultivated in ADaM medium with the yeast $S$. cerevisiae as a food substrate served as the control; this is the traditional
Table 1

The scheme of experiment with synthetic $\mathrm{ADaM}$ or water from recirculating aquaculture systems (RAS) and $S$. cerevisiae, $R$. glutinis, or R. rubra as food substrate for M. macrocopa

\begin{tabular}{lll}
\hline \hline $\begin{array}{l}\text { Experimental } \\
\text { group }\end{array}$ & Medium & Food substrate \\
\hline \hline I & ADaM & S. cerevisiae \\
II & ADaM & $R$. glutinis \\
III & ADaM & $R$. rubra \\
IV & Water from RAS & S. cerevisiae \\
V & Water from RAS & $R$. glutinis \\
VI & Water from RAS & $R$. rubra \\
\hline \hline
\end{tabular}

biotechnology used in zooplankton cultivation (Chakri et al. 2014, Khatun et al. 2014). Yeast suspension quantities of $24 \times 10^{6}$ cells per 11 of medium in which M. macrocopa was cultivated were applied every 48 hours. Yeasts $R$. glutinis and $R$. rubra were cultivated in a pepted meat broth. The inoculum was obtained by growing a seed culture for 2 days at $27^{\circ} \mathrm{C}$. Spectrophotometric measurements of the suspension's optical density were done at $\lambda=625 \mathrm{~nm}$ to adjust the concentration of the microorganisms to $1.5 \times 10^{8} \mathrm{CFU} \mathrm{m}^{-1}$. Seed yeast was cultivated in an LOIP LS-110 (160 rpm) laboratory shaker at $25-27^{\circ} \mathrm{C}$ for five days. The microbial suspension was centrifuged for $20 \mathrm{~min}$. at $1500 \mathrm{~g}$ to separate the cultivated broth from the yeast biomass. The yeast cells were counted using a Goryaev camera, while the crustaceans were counted using the aliquot method with a Bogorov camera under a MicroMed XS-3300 binocular microscope. The dynamics of the basic physicochemical parameters of the medium were measured during M. macrocopa cultivation. Conductivity and total mineralization in the culture medium was determined with an SX-650 (Ulab) conductivity meter. Total carotenoids in M. macrocopa were determined using conventional methods (GOST R 2011).

Two-way ANOVA was used to analyze differences in the growth of the M. macrocopa culture and total carotenoid content, and the degree of total mineralization and conductivity of the culture media with different food substrates. Mean values were considered significantly different at $\mathrm{P} \leq 0.05$. The results were analyzed statistically with Microsoft Excel software. 


\section{Results}

Culture growth decelerated until it stopped completely on the fourth day of the experiment with yeast $S$. cerevisiae as the food substrate for M. macrocopa in ADaM. In contrast, the RAS post-production water cultivation medium ensured rapid culture growth on the same food substrate. When the crustaceans were fed the two investigated species of carotenogenic yeast, the influence of cultivation medium on culture growth dynamics was negligible (Fig. 1). Two-way ANOVA indicated significant differences among all the investigated groups on the second, sixth, and eighth days of cultivation. No significant differences among all the experimental groups were found on the fourth day of the experiment.

The analysis of the physicochemical parameters of both culture media indicated a general tendency for a gradual increase in conductivity and total mineralization (Fig. 2). No significant differences in conductivity and total mineralization values of either media studied were observed. Using carotenogenic yeasts as food substrates stimulated the accumulation of carotenoids in $M$. macrocopa cultures (Fig. 3). The maximum carotenoid values (14 $\mathrm{mg} \mathrm{g}^{-1}$ dry matter) were achieved when $M$. macrocopa was cultured using $R$. glutinis in ADaM medium. Maximum carotenoid levels were also observed on the sixth day of cultivation when the crustaceans were cultured with $R$. glutinis in RAS water. The efficiency of carotenoid accumulation was twice as high in M. macrocopa cultivated in the synthetic AdaM medium. In all experimental groups in which carotenogenic yeast was used, the level of accumulated carotenoids in M. macrocopa decreased almost to the baseline value by the eighth day of cultivation. Significant differences in carotenoid content among all experimental groups were observed on each day of cultivation. It should be noted that crustacean cultures fed on $R$. glutinis accumulated more carotenoids in both medium types; however, carotenoid saturation was more rapid when the crustaceans were cultured in ADaM.

\section{Discussion}

Continuous cultivation without cleaning or upgrading the culture medium is accompanied by the accumulation of the final products of metabolism. The increased conductivity and total mineralization observed in the current experiment is an indirect indication of this (Fig. 2a). The accumulation of toxic metabolites in cultivation medium can induce free
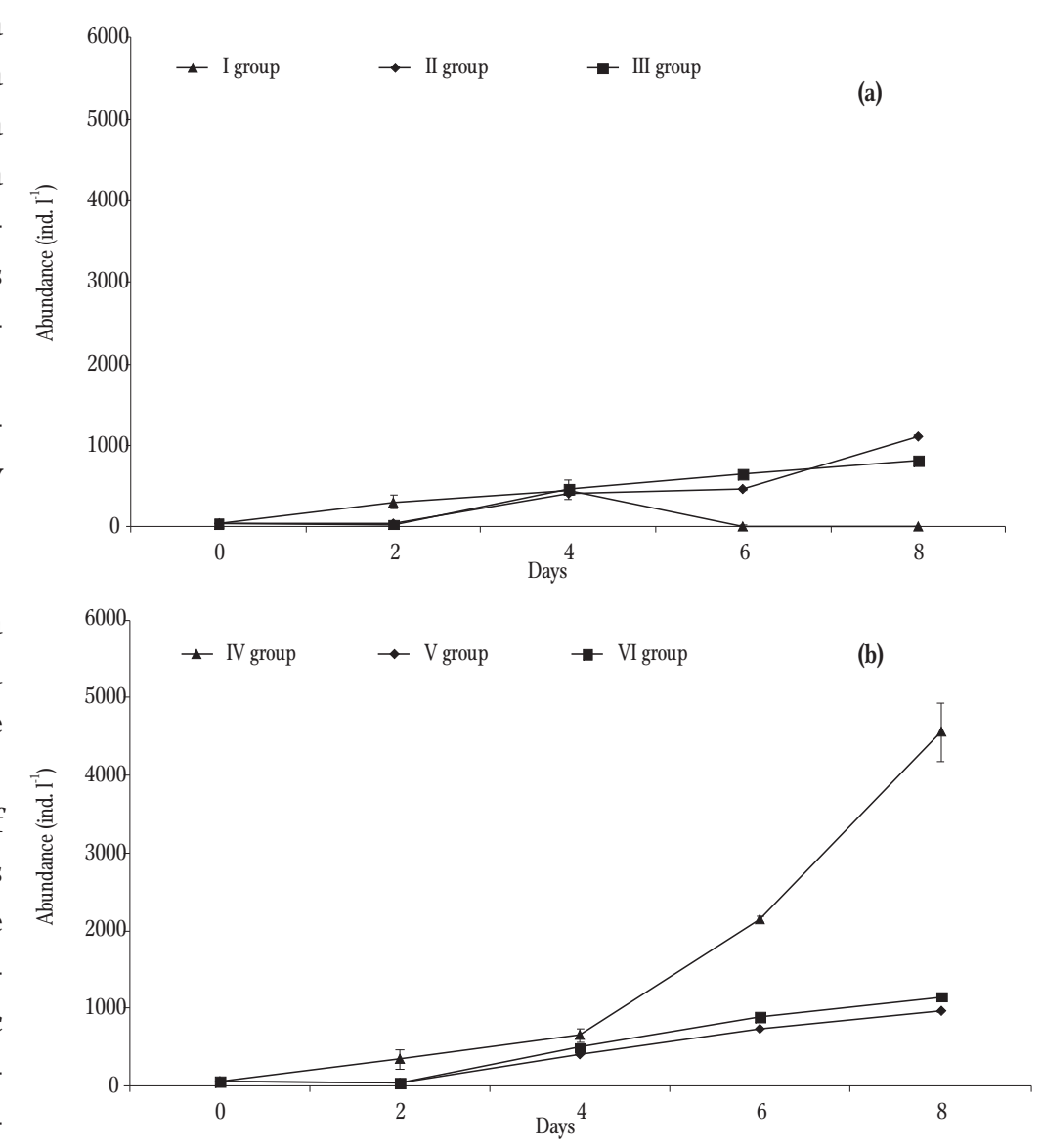

Figure 1. Growth dynamics of M. macrocopa cultures during cultivation in two different media (a - ADaM; b - RAS post-production water). Note: groups I and IV fed $S$. cerevisiae, groups II and V fed R. glutinis, groups III and VI fed R. rubra. 

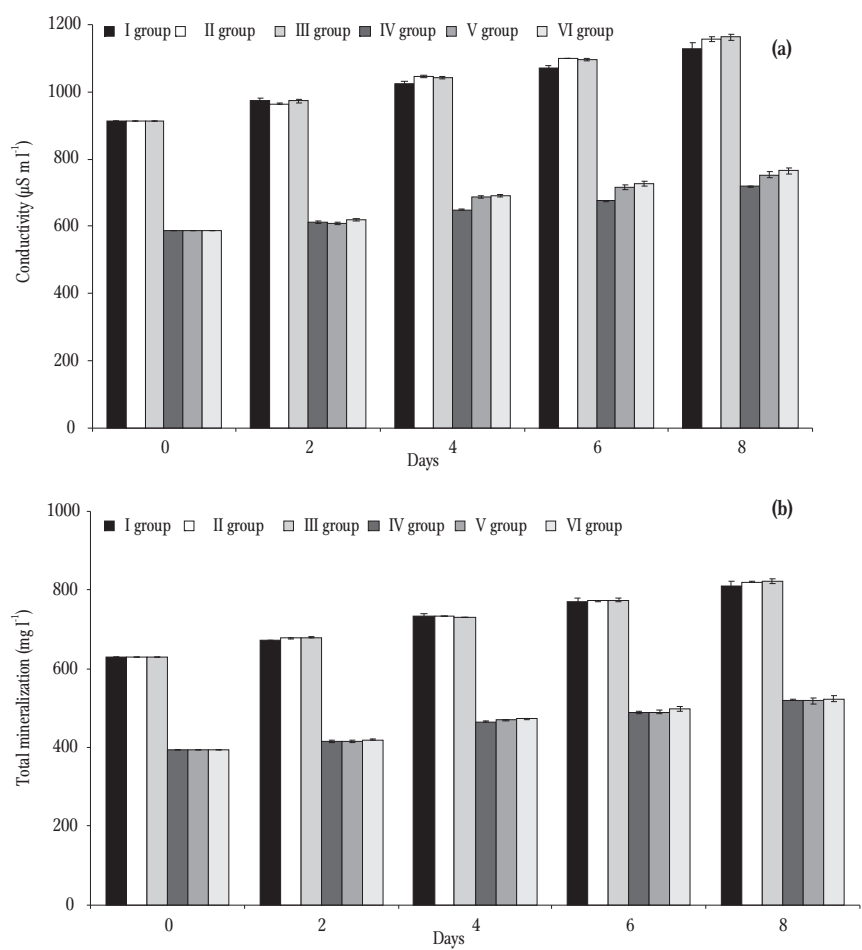

Figure 2. Dynamics of a) conductivity $\left(\mu \mathrm{S} \mathrm{ml}^{-1}\right)$ and b) total mineralization $\left(\mathrm{mg} \mathrm{l}^{-1}\right)$ during cultivation in different media ( $\mathrm{a}-\mathrm{ADaM}$; $\mathrm{b}$ - RAS post-production water) with different yeasts. Note: groups I and IV fed $S$. cerevisiae, groups II and V fed $R$. glutinis, groups III and VI fed R. rubra.
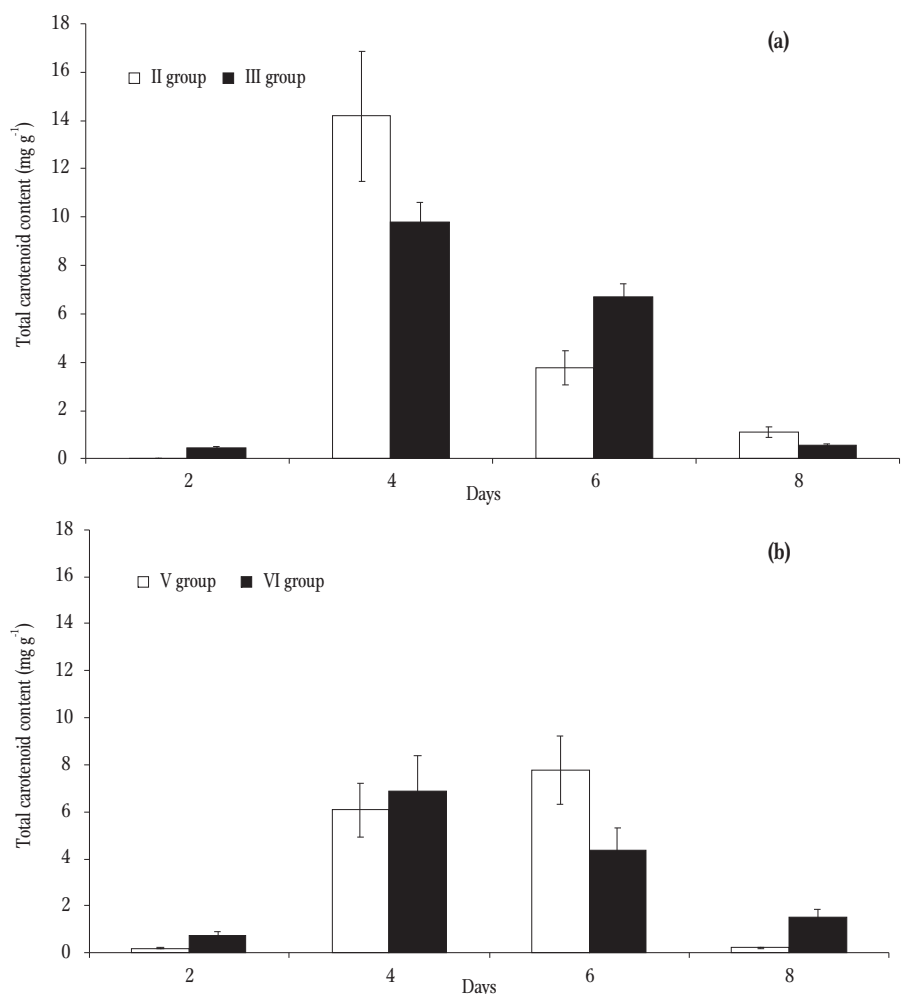

Figure 3. Total carotenoid content in M. macrocopa during cultivation in two different media (a - ADaM; b - RAS post-production water) using yeasts $R$. glutinis and $R$. rubra. Note: groups II and V fed $R$. glutinis, groups III and VI fed $R$. rubra. radical oxidation processes. The activation of lipid peroxidation and oxidative modification of proteins are linked with the excessive formation of free radicals that is accompanied by the contravention of biological membrane properties and cell function in general. Obviously, these processes inhibit the control culture of M. macrocopa after the fourth day of cultivation (Fig. 1a).

The role of carotenoids as antioxidants is well known (Shmarakov et al. 2013). The lack of degradation and even slight increases in numbers in the cultures that received Rhodotorula yeast food substrate instead of $S$. cerevisiae (Fig. 1a) can be explained by the accumulation of carotenoids in $M$. macrocopa (Fig. 3). Crustaceans store carotenoids in the intestinal walls (Herring 1968, Pandolfini et al. 2000), fat cells, and eggs, and sometimes in the maxillary glands and blood, but they are absent in the epidermis and cuticle. The quantitative characteristics of carotenoids in crustaceans depend on the type of food substrate and the physiological state of the organisms. Thus, increased carotenoid content is observed during maturation and egg production (Green 1957).

The carotenoid content in $M$. macrocopa fed yeast $R$. glutinis was approximately 1.5 times higher than that in the crustaceans fed $R$. rubra. This is obviously because $R$. glutinis exhibited much higher carotenogenic activity than did R. rubra (Kiritsa 2005). After reaching the maximum content in $M$. macrocopa, the carotenoids are not stable and begin to decrease even though the crustaceans continued to receive carotenogenic yeast as food. This could have been caused by carotenoid involvement in antioxidant system 
activation mechanisms that protect the body from the free radical oxidation of biomolecules caused by stress induced by the deterioration of the culture medium. The replacement of ADaM with RAS post-production water did not lead to a deterioration in the growth dynamics of $M$. macrocopa cultures (Fig. 1b) because all the necessary components for normal crustacean development are in RAS post-production water from fish food. Using RAS post-production water can reduce the cost of live feed cultivation technology in the future.

\section{Conclusions}

Replacing the standard ionic composition medium AdaM with RAS post-production water for the cultivation of M. macrocopa does not result in any deterioration in the intensity of the growing crustacean culture.

Using the carotenogenic yeast Rhodotorula as a food substrate improves the carotenoid content in M. macrocopa by the fourth to sixth days of cultivation. Prolonged M. macrocopa cultivation with carotenogenic yeast is inappropriate.

Author contributions. O.Kh. and L.K. designed the research; O.Ku. performed the research; O.Ku., O.Kh., L.K., R.K. and M.M. analyzed the data; O.Ku., O.Kh., L.K., R.K. and M.M. wrote the paper.

\section{References}

Bjerkeng B. 2000 - Carotenoid pigmentation of salmonid fishes recent progress - In: Avances en Nutricion Acuicola V. Memorias del V Simposium Internacional de Nutricion Acuicola (Eds) L.E. Cruz-Suarez, D. Ricque-Marie, M. Tapia-Salazar, M.A. Olvera-Novoa, R. Civera-Cerecedo, Universidad Autónoma de Nuevo León, Monterrey, N.L. México: 71-89.

Buzzini P., Martini A. 1999 - Production of carotenoids by strains of Rhodotorula glutinis cultured in raw materials of agro-industrial origin - Bioresour. Technol. 71: 41-44.

Chakri K., Berrak H., Samraoui B. 2014 - Effect of food concentration on the development, growth, reproduction and total life span of Simocephalus expinosus Koch (Cladocera: Daphniidae) - Annal. Biol. Res. 5: 55-58.
Czeczuga B. 1988 - Carotenoids in fish. Cyprinidae: Aspius aspius (L.) - Acta Ichthyol. Piscat. 18: 97-102.

Czeczuga B. 1994 - Carotenoids in fish. 51. Cyprinidae Phytophagus: Rutilus rutilus, Rutilus rutilus heckeli, Scardinus erythrophtalmus, Chondrostoma nasus and Rhodeus sericeus amarus - Acta Ichthyol. Piscat. 24: 43-51.

Czeczuga B., Dabrowski K., Rosch R., Champigneulle A. 1991 - Carorenoids in fish. 48 Carotenoids in Coregonus lavaretus L. individuals of various populations - Acta Ichthyol. Piscat. 21: 3-16.

Das P., Mandal S.C., Bhagabati S.K., AkhtarM.S., SinghS.K. 2012 - Important live food organisms and their role in aquaculture - In: Frontiers in Aquaculture (Ed.) M. Sukham, Narendra Publishing House: 69-86.

Faruque M.M., Kawser A. Md., Quddus M.M.A. 2010 -Use of live food and artificial diet supply for the growth and survival of African catfish (Clarias gariepinus) larvae World J. Zool. 5: 82-89.

GOST R 2011 - Functional Food. Method for determination of carotinoids. GOST R 54058-2010 - Standartinform Moscow, Russia, 8 p. (in Russian).

Green J. 1957 - Carotenoids in Daphnia - Proc. R. Soc. B-Biol. Sci. 147: 392-401.

Guedes A.K., Amaro H.M., Malcata F.X. 2011 - Microalgae as sources of carotenoids - Mar. Drugs. 9: 625-644.

Gupta S.K., Jha A.K., Pal A.K., Venkateshwarlu G. 2007 Use of natural carotenoids for pigmentation in fishes Natural Product Radiance 6: 46-49.

Herring P.J. 1968 - The carotenoid pigments of Daphnia magna Straus - I. The pigments of animals fed Chlorella pyrenoidosa and pure carotenoids - Comp. Biochem. Physiol. 24: 187-203.

Khatun B., Rahman R., Rahman M.S. 2014 - Evaluation of yeast Saccharomyces cerevisiae and algae Chlorella vulgaris as diet for rotifer Brachionus calyciflorus - The Agriculturists 12: 1-9.

Kiritsa E. 2005 - Directed synthesis of carotenoids in yeast, and the prospect of their using - Ph.D. thesis, Institute of Microbiology, NAN Moldova, Chisinau, Moldova, 129 p. (in Russian).

Kluttgen B., Dulmer U., Engels M., Ratte H.T. 1994 - ADaM, an artificial freshwater for the culture of zooplankton Water Res. 28: 743-746.

Nechypurenko O., Avdeeva L., Kharkhota M. 2013 - Carotine synthesizing abilities and probiotic properties of bacteria of Bacillus genus - Naukovi zapisky 142: 25-35 (in Ukrainian).

Pandolfini E., Thys I., Leporcq B., Descy J.-P. 2000 - Grazing experiments with two freshwater zooplankters: fate of chlorophyll and carotenoid pigments - J. Plankton Res. 22: 305-319.

Romanenko V.D., Krot Yu. G., Syrenko L.A., Solomatina V.D. 1999 - The biotechnology of hydrobionts cultivation - 
Institute of Hydrobiology of NAS of Ukraine, Kyiv, Ukraine, 264 p. (in Ukrainian).

Shmarakov I.O., Yuen J.J., Blaner W.S. 2013 - Carotenoid metabolism and enzymology - In: Carotenoids and
Human Health (Ed.) S.A. Tanumihardjo, Springer Science and Business Media, Humana Press: 28-56.

Wozniak M. 1996 - The role of carotenoids in fish - Acta Acad. Agricult. Tech. Olst. 22: 65-74 (in Polish). 